DRAFT VERSION JULY 4, 2018

Preprint typeset using $\mathrm{LT}_{\mathrm{E}} \mathrm{X}$ style emulateapj v. 6/22/04

\title{
HAS THE BLACK HOLE IN XTE J1118+480 EXPERIENCED AN ASYMMETRIC NATAL KICK?
}

\author{
Alessia Gualandris ${ }^{1}$, Monica Colpi ${ }^{2}$, Simon Portegies Zwart ${ }^{1}$ And Andrea Possenti ${ }^{3}$ \\ Draft version July 4, 2018
}

\begin{abstract}
We explore the origin of the Galactic high latitude black hole X-ray binary XTE J1118+480, and in particular its birth location and the magnitude of the kick received by the black hole upon formation in the supernova explosion. Our analysis is constrained by the evolutionary state of the companion star, the observed limits on the orbital inclination, the Galactic position and the peculiar velocity of the binary system. We constrain the age of the companion to the black hole using stellar evolution calculations between $2 \mathrm{Gyr}$ and $5 \mathrm{Gyr}$, making an origin in a globular cluster unlikely. We therefore argue that the system was born in the Galactic disk, in which case the supernova must have propelled it in its current high latitude orbit. Given the current estimates on its position in the sky, proper motion and radial velocity, we back-trace the orbit of XTE J1118+480 in the Galactic potential to infer the peculiar velocity of the system at different disk crossings over the last 5 Gyr. Taking into account the uncertainties on the velocity components, we infer that the peculiar velocity required to change from a Galactic disk orbit to the currently observed orbit is $183 \pm 31 \mathrm{~km} \mathrm{~s}^{-1}$. The maximum velocity which the binary can acquire by symmetric supernova mass loss is about $100 \mathrm{~km} \mathrm{~s}^{-1}$, which is $2.7 \sigma$ away from the mean of the peculiar velocity distribution. We therefore argue that an additional asymmetric kick velocity is required. By considering the orientation of the system relative to the plane of the sky, we derive a $95 \%$ probability for a non null component of the kick perpendicular to the orbital plane of the binary. The distribution of perpendicular velocities is skewed to lower velocities with an average of $93_{-60}^{+55} \mathrm{~km} \mathrm{~s}^{-1}$. These estimates are independent of the age of the system, but depend quite sensitively on the kinematic parameters of the system. A better constraint on the asymmetric kick velocity requires an order of magnitude improvement in the measurement of the current space velocity of the system.
\end{abstract}

Subject headings: stars: black-holes - stars: individual (XTE J1118+480) - stars: binary - X-ray

\section{INTRODUCTION}

The velocity dispersion of the low mass X-ray binaries hosting a black hole (Liu, van Paradijs \& van den Heuvel 2001 ) is around $40 \mathrm{~km} \mathrm{~s}^{-1}$, as derived by White \& van Paradijs (1996) from a study of the vertical distribution of these systems in the Galaxy. Since the velocity dispersion of the population of their progenitors (i.e. binary systems containing a star more massive than $\sim 20 \mathrm{M}_{\odot}$, Portegies Zwart, Verbunt, Ergma 1997) is estimated to be $\sim 20 \mathrm{~km} \mathrm{~s}^{-1}$ (Mihalas \& Binney 1981), there is evidence for the acquisition of an extra velocity during the formation of the black hole. There are two possible mechanisms to accelerate a binary system when a massive star forms and explodes as a supernova. The first is the ejection of mass from the binary (Blaauw 1961). The released mass continues to move with the velocity of its center of mass and, for conservation of linear momentum, the binary recoils in the opposite direction. We will refer to this velocity acquired by the binary as symmetric kick. The direction of the symmetric kick lies in the orbital plane of the binary which means that the direction of the orbital angular momentum vector of the system is conserved by the explosion. The second mechanism is known as asymmetric kick and represents an additional kick generated by asymmetries seeded in the collapsing core that are transmitted to the ejecta. These natal kicks can have random directions relative to the orbital plane. There is evidence for the occurrence of asymmetric

1 University of Amsterdam, Kruislaan 403, 1098 SJ, Amsterdam, the Netherlands

${ }^{2}$ Università degli Studi di Milano Bicocca, Piazza della Scienza 3, 20100 Milano, Italy

${ }^{3}$ INAF-Osservatorio Astronomico di Cagliari, loc. Poggio dei Pini, Strada 54, 09012, Capoterra, Italy kicks in the formation of neutron stars (see Lai, Chernoff \& Cordes 2001 for a review) but the physical interpretation is still controversial: mechanisms proposed include large scale density asymmetries in the pre-supernova core (Burrows \& Hayes 1996), or non-axisymmetric instabilities in the rapidly rotating proto-neutron star core (Colpi \& Wasserman 2002). These hydro-dynamically driven impulses can in principle operate at the time of black hole formation. This is expected in particular if a hot proto-neutron star forms first, launching a successful shock wave, and later collapses to a black hole due to falling back material (Heger et al. 2000). The highest neutron star kick recorded is of $800-1600 \mathrm{~km} \mathrm{~s}^{-1}$ (Cordes, Romani \& Lundgren 1993) so if kicks scale approximately with the inverse of the mass of the compact object, peculiar velocities as large as $100 \mathrm{~km} \mathrm{~s}^{-1}$ are expected for black holes. The first discovered runaway black hole is in the binary X-Ray Nova GRO J1655-40 (Mirabel et al. 2002) for which there is evidence of a motion of $112 \pm 18 \mathrm{~km} \mathrm{~s}^{-1}$. GRO J1655-40 is a source that shows alpha-elements in its optical spectrum indicating that a supernova explosion preceded the formation of the black hole (Israelian et al. 1999).

In this work we focus on XTE J1118+480, a soft X-ray transient (i.e. a low-mass X-ray binary exhibiting recurrent bright optical and X-ray outbursts, often accompanied by radio activity, Tanaka \& Shibazaki 1996, Campana et al. 1998) with a mass function large enough $\left(f(M) \approx 6.0 \pm 0.4 \mathrm{M}_{\odot}, \mathrm{Mc}-\right.$ Clintock et al. 2001) to ensure it hosts a black hole. Three features distinguish XTE J1118+480 in the sample of 14 similar systems (Lee, Brown \& Wijers, 2002; Orosz et al. 2002): (i) the high-galactic latitude $\left(l=157.7^{\circ}, b=62.3^{\circ}\right.$, corresponding to a distance of $1.9 \pm 0.4 \mathrm{kpc}$ from the Sun with a height of $1.7 \pm 0.4 \mathrm{kpc}$ above the Galactic plane, Wagner et al. 2001); (ii) the high space velocity $\left(\sim 145 \mathrm{~km} \mathrm{~s}^{-1}\right.$ rela- 
tive to the Local Standard of Rest, Mirabel et al. 2001, much larger than the aforementioned velocity dispersion of the population); (iii) the shortest orbital period $P=4.08$ hours (Cook et al. 2000). Optical photometry (Wagner et al. 2001) suggests that the donor is $\mathrm{a} \sim 0.3 \pm 0.2 \mathrm{M}_{\odot}$ star now filling its Roche lobe of $\sim 0.35 \mathrm{R}_{\odot}$. Modeling of the light curve (McClintock et al. 2001) indicates that the inclination of the system is high, $i \gtrsim 55^{\circ}$, and the mass of the black hole is consequently modest, $M_{\mathrm{bh}} \lesssim 10 \mathrm{M}_{\odot}$. Additional evidence for a high inclination $\left(i \gtrsim 60^{\circ}\right)$ comes from measurements of tidal distortion (Frontera et al. 2001) whereas the lack of dips or eclipses for a Roche-lobe filling secondary yields upper limits of $i<80^{\circ}$ and $M_{\mathrm{bh}} \gtrsim 7.1 \mathrm{M}_{\odot}$. The extremely low hydrogen column depth $\left(N_{\mathrm{H}} \approx 1.3 \times 10^{20} \mathrm{~cm}^{-2}\right)$ and the modest distance make this system a primary target for the study of the origin and the dynamics of Galactic black holes.

Mirabel et al. (2001) noticed that the orbit of XTE J1118+ 480 resembles that of halo objects like Galactic globular clusters. In addition, the radial and azimuthal components of the velocity seem consistent with the large random motions of old halo stars with low metallicities. According to these characteristics, Mirabel et al. suggested that the black hole in XTE J1118+480 may be one of the black holes ejected from globular clusters which are believed to swirl around in the halo of the Galaxy. This hypothesis requires the binary system to be coeval with the globular clusters.

In this paper we exploit recent results (Haswell et al. 2002) on the evolution of XTE J1118+480, which hint for a much younger age of the system. We show that an ejection from a globular cluster is unlikely $(\S 2)$ and propose that the system originated in the disk of the Galaxy and was then launched at high latitude by a large kick induced by the supernova explosion. In $\S 3$ we investigate the nature of this kick, by backtracking the orbit of XTE J1118+480 in the Galactic potential in order to derive the distribution of the runaway velocity and of its component perpendicular to the orbital plane, signature of an asymmetric explosion in the formation of the black hole. In $\S 4$ we summarize our conclusions.

\section{THE BIRTHPLACE OF THE SYSTEM}

Spectroscopic studies of XTE J1118 + 480 provide important clues about the nature of the system and the evolution of its orbital parameters. The UV spectrum of XTE J1118+480 (Haswell et al. 2002) show evidence of an under-abundance of carbon compared to nitrogen which suggests that the material accreted by the black hole has been substantially CNOprocessed. This implies that the companion star has lost its outer layers and is now exposing inner regions which have been enriched with CNO-processed material from the central core. Haswell et al. interpret these qualitative observations as an indication that the system came into contact when the companion star was sufficiently massive to allow for the CNO cycle and was already somehow evolved. These requirements constrain the orbital period at contact $P_{i}$ to be longer than about 12 hours and the companion mass to be about $1.5 \mathrm{M}_{\odot}$. The evolution of the system after contact was driven by angular momentum losses, mainly magnetic braking, toward shorter orbital periods. The estimate of the initial mass of the companion star is crucial to this investigation as it allows a determination of an upper limit for the age of the system. The latter can in turn constrain the system's birthplace to be the Galactic disk or a globular cluster.

Supernova explosions of massive stars occur in the first $10^{7}$ years since the formation of a globular cluster, producing neu- tron stars and black holes. The age of XTE J1118+480 is hence determined by the main sequence lifetime of the companion star and by the binary evolution phase after contact. A $1.5 \mathrm{M}_{\odot}$ star has a main sequence lifetime $T_{\mathrm{MS}} \sim 2 \mathrm{Gyr}$. The duration of the mass transfer phase can be estimated by means of binary evolution calculations. Using the code by Eggleton (Pols et al. 1995), J.Dewi (private communication) followed the evolution of a system composed by a black hole of $7 \mathrm{M}_{\odot}$ and a main sequence companion of $1.4 \mathrm{M}_{\odot}$ with an initial period $P_{i}=15$ hours. The binary shrinks, reaching the period $(P=4.08$ hours) currently observed in XTE J1118 +480 after a time $T_{\mathrm{ev}} \sim 0.8 \mathrm{Gyr}$ (Fig. 1 reports the evolution of the mass of the companion star as a function of the binary orbital period). An upper limit for the age of the system can then be determined as

$$
\tau=T_{\mathrm{MS}}+T_{\mathrm{ev}} \sim 3 \mathrm{Gyr} .
$$

If, according to the uncertainties in the evolutionary models, we allow for a range of initial masses of the companion star $1.2 \mathrm{M}_{\odot} \lesssim m \lesssim 1.8 \mathrm{M}_{\odot}$, the main sequence lifetime for the companion is limited to the interval $1 \mathrm{Gyr} \lesssim \mathrm{T}_{\mathrm{MS}} \lesssim 4.5 \mathrm{Gyr}$, and, as a consequence, the age of the system must lie in the range $2 \mathrm{Gyr} \lesssim \tau \lesssim 5.5 \mathrm{Gyr}$. On the basis of these estimates we can exclude that the system was formed and then ejected from one of the known Galactic globular clusters, which halted forming stars more than $10 \mathrm{Gyr}$ ago ${ }^{4}$.

There exist further uncertainties associated with the evolution of XTE J1118+480 which may make somehow affect the estimate of $\tau$. In particular, differences in the modeling of magnetic braking can introduce a scatter in the value of $\tau$ : a weaker braking would cause the binary to harden up to the semi-detached state on a longer time. Other uncertainties are related to the observed properties of the companion star, like the luminosity, effective temperature and metallicity, which may affect the estimate of the age of the system. A quantitative evaluation of such uncertainties would require detailed studies which are beyond the aim of this work. Nonetheless, conciliating $\tau$ with the hypothesis of the origin of the binary in a globular cluster would demand an unlikely fine-tuning of the binary evolution. In this paper we assume a standard model for the binary evolution, like the one adopted by Haswell et al. (2002), according to which XTE J1118+480 is too young to have been ejected from a globular cluster ${ }^{5}$ and hence we conclude that the system was very likely born in the disk of the Galaxy.

\section{THE SUPERNOVA KICK HYPOTHESIS: BACK-TRACKING THE ORBIT OF XTE J1118+480 IN THE GALACTIC POTENTIAL}

We now consider the hypothesis that the system originated in the disk of the Galaxy and was launched at high galactic latitude by a kick induced by the supernova explosion which pro-

\footnotetext{
4 There exists a possibility that the black hole in XTE J1118 + 480 experienced a dynamical encounter in a globular cluster with a blue straggler in a binary system tight enough to lead to the ejection of the black hole and the newly acquired companion. Stellar encounters involving black holes are effective in the first billion year since the cluster formation (Portegies Zwart \& McMillan 2000) as the black holes tend to interact with each other, form binaries which harden through subsequent interactions and eject each other from the cluster (Sigurdsson \& Hernquist 1993; Kulkarni, Hut, McMillan 1993). This scenario requires that at least one black hole is retained and that blue stragglers are continuously formed in the core, preferentially in a hard binary. We consider this possibility very unlikely.

5 The only scenario compatible with an origin in a cluster is that of a black hole ejected $\sim 10 \mathrm{Gyr}$ ago from a cluster and then capable of capturing a main sequence star of about $1.5 \mathrm{M}_{\odot}$ while wandering through the Galaxy: the probability for such an event in the low stellar density of the Galaxy is in fact negligible.
} 


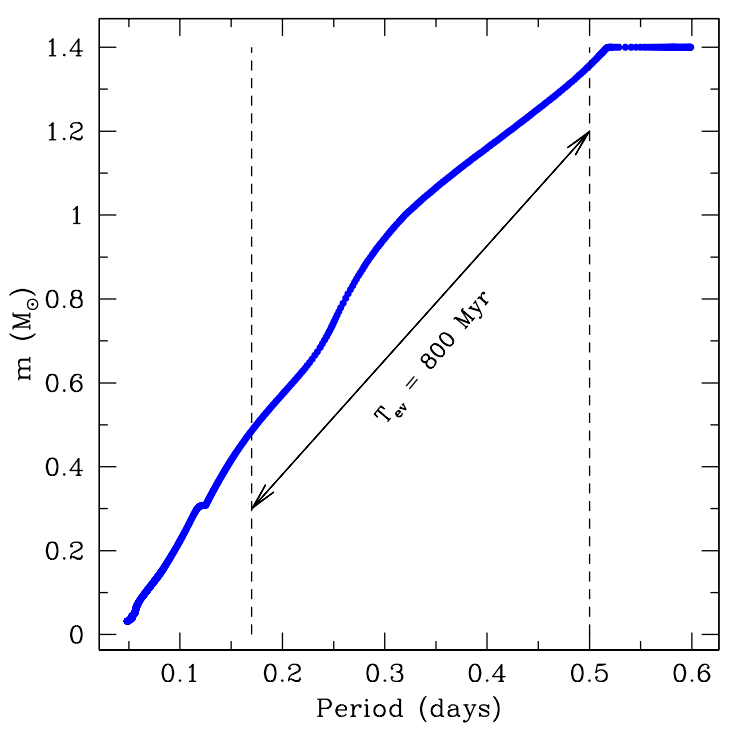

FIG. 1.- Evolution of a binary system composed by a black hole of $7 \mathrm{M}_{\odot}$ and a main sequence star of $1.4 \mathrm{M}_{\odot}$ with an orbital period at first contact $P_{i}=0.5$ days. Angular momentum losses drive the shrinking of the binary until, after a time $T_{\mathrm{ev}} \simeq 800 \mathrm{Myr}$, the system reaches the current period of about 0.17 days. At that point the companion star has a mass of about $0.48 \mathrm{M}_{\odot}$, compatible with the observed mass $m=(0.3 \pm 0.2) \mathrm{M}_{\odot}$ of the donor in XTE J1118+480.

duced the black hole. Given the large mass of the black hole progenitor, its evolution must have been very fast, whence we can assume that the supernova event took place shortly after the beginning of the main sequence life of the donor star. However, the time of expulsion $\tau_{\text {ex }}$ from the Galactic disk is affected by the uncertainties in the duration of the X-ray phase and in the mass interval of the donor star. The length of the $\mathrm{X}$-ray phase determines a lower limit of $0.5-1$ Gyr since mass transfer necessarily begins after the black hole formation. On the other hand, an upper limit of 2-5 Gyr reflects the age of the companion star on the main sequence. More specifically, the lower and upper values refer to a star which is now respectively at the beginning or at the end of its main sequence phase. Combining these limits, for the most probable mass of the donor star $m=1.5 \mathrm{M}_{\odot}$ (Haswell et al. 2002) we obtain $0.8 \lesssim \tau_{\text {ex }} \lesssim 3$ Gyr.

\subsection{Integration of the galactic orbit of XTE J1118+480 backward in time}

The integration of the trajectory of XTE J1118 + 480 backward in time provides the value of the kick velocity acquired at birth by the black hole when residing in the Galactic disk. It is our aim to compute this value which is nonetheless affected by a number of uncertainties. The orbit of the system is in fact not unique given the finite interval of expulsion times and the observational errors in the velocity vector. The observed proper motion and the radial velocity are known with $20 \%$ and $50 \%$ accuracy, respectively. Thus, a statistical approach is necessary for the study of the orbit. We generate the initial conditions for the integration by drawing at random the three components of the velocity vector from Gaussian dis- tributions. Once we have initialized the velocity components for 10000 different Monte Carlo realizations, we trace the trajectories backward in time with a fifth order Runge-Kutta integrator using the Paczynski model (Paczynski 1990) for the potential of the Galaxy.

In Figure 2 we show the position of the source in the Galactic plane obtained integrating up to a time $0.5 \mathrm{Gyr}$ (left panel) and, continuing the integration, up to $5 \mathrm{Gyr}$ (right panel). We notice that, due to the uncertainties in the current 3D velocity, memory is lost of the position of the binary at the time of formation, if the explosion occurred more than $1 \mathrm{Gyr}$. (We verified that a change of $10 \%$ in the parameters of the Galactic potential results in differences of at most $5 \%$ in the velocity of the system.)

Figure 3 shows the distributions of the modulus of the peculiar velocity of the system (after correcting for rotation around the Galactic center) obtained from the Monte Carlo realizations corresponding to the passages through the disk closer to some specified time. In particular, we select the crossings closest to 0.5, 2, 3 and 5 Gyr in the past. The distributions for the peculiar velocity tend to widen while increasing time backward, but the mean and the shape of the distributions do not change considerably at the different passages through the disk, allowing to safely adopt as reference value for the peculiar velocity the average computed over all the disk crossings between 0.5 and 5 Gyr ago, $\left\langle V_{p}\right\rangle=183 \pm 31 \mathrm{~km} \mathrm{~s}^{-1}$.

We first explore $(\S 3.2)$ the possibility that a symmetric kick is entirely responsible for the large peculiar velocity of XTE J1118+480 and show that this scenario is very unlikely. In $\S 3.3$ we combine the available information on the $3 \mathrm{D}$ orientation of the binary in the celestial sphere and the results on its dynamics through the Galaxy to put constraints on the contribution from an additional asymmetric kick, intrinsic to the supernova event.

\subsection{Symmetric kick}

The amount of mass $\Delta M$ which has to be ejected during a symmetric supernova explosion in order to produce a kick velocity $V_{\text {sym }}$ can be written as a function of the black hole mass $M_{\mathrm{bh}}$ and the companion mass $m$ (Bhattacharya $\&$ van den Heuvel, 1991; Nelemans, Tauris, van den Heuvel 1999) as

$$
\left(\frac{\Delta M}{\mathrm{M}_{\odot}}\right)=\left(\frac{V_{\mathrm{sym}}}{213 \mathrm{~km} \mathrm{~s}^{-1}}\right)\left(\frac{P}{\text { day }}\right)^{\frac{1}{3}}\left(\frac{M_{\mathrm{bh}}+m}{\mathrm{M}_{\odot}}\right)^{\frac{5}{3}}\left(\frac{\mathrm{M}_{\odot}}{m}\right)
$$

where $P$ is the orbital period of the circularized binary after the explosion. The system remains bound after the supernova only if the amount of ejected mass is smaller than half the total mass of the system before the explosion, $\Delta M<0.5\left(m+M_{\mathrm{He}}\right)$, where we indicate with $M_{\mathrm{He}}$ the mass of the black hole progenitor. If we replace $M_{\mathrm{He}}=M_{\mathrm{bh}}+\Delta M$, the above condition can be written as $\Delta M<\left(M_{\mathrm{bh}}+m\right)$.

The peculiar velocity of the system at the most recent disk crossing, as determined by Mirabel et al. (2001), is $V_{p}=217 \pm$ $18 \mathrm{~km} \mathrm{~s}^{-1}$. They used this value of the velocity together with the current mass $\left(\sim 0.3 \mathrm{M}_{\odot}\right)$ of the companion star and the current orbital period to estimate the ejected mass needed in a symmetric supernova explosion. They found that more than $40 \mathrm{M}_{\odot}$ should have been ejected during the stellar collapse in order to accelerate the black hole system up to the velocity $V_{p}$. This value is implausibly large for the binary to remain bound.

In order to improve on that calculation, we have inte- 

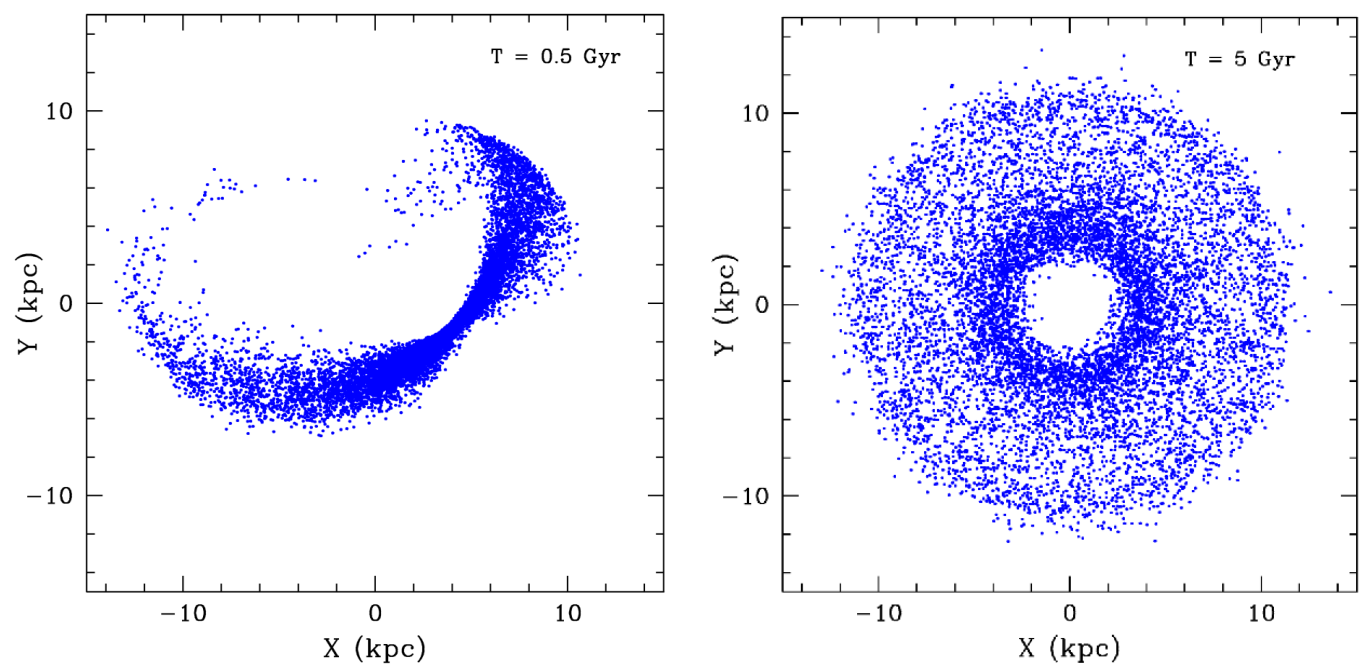

FIG. 2.- Position of XTE J1118 +480 in the Galactic plane (the Sun being at a position X=-8.5 kpc, Y=0) at an epoch of $0.5 \mathrm{Gyr}$ ago (left plot) and $5 \mathrm{Gyr}$ ago (right plot) obtained from 10000 integrations of its trajectory backward in time starting from its current position $\left(l=157.7^{\circ}, \delta=+62.3^{\circ}, d=1.85 \mathrm{kpc}\right)$. The velocity components are randomly drawn from Gaussian distributions $\left(U=-105 \pm 16 \mathrm{~km} \mathrm{~s}^{-1}, V=+122 \pm 16 \mathrm{~km} \mathrm{~s}^{-1}, W=-21 \pm 10 \mathrm{~km} \mathrm{~s}^{-1}\right.$, relative to the Galactic center) using the Monte Carlo code described in $\$ 3.1$. The uncertainties on the current 3D velocity of the system propagate throughout the integration with a resulting loss of information on the position of the system at any time in the past anterior to about $1 \mathrm{Gyr}$ ago. However, the shape of the orbit is such that the system always remains at a distance greater than about $2 \mathrm{kpc}$ from the Galactic center.
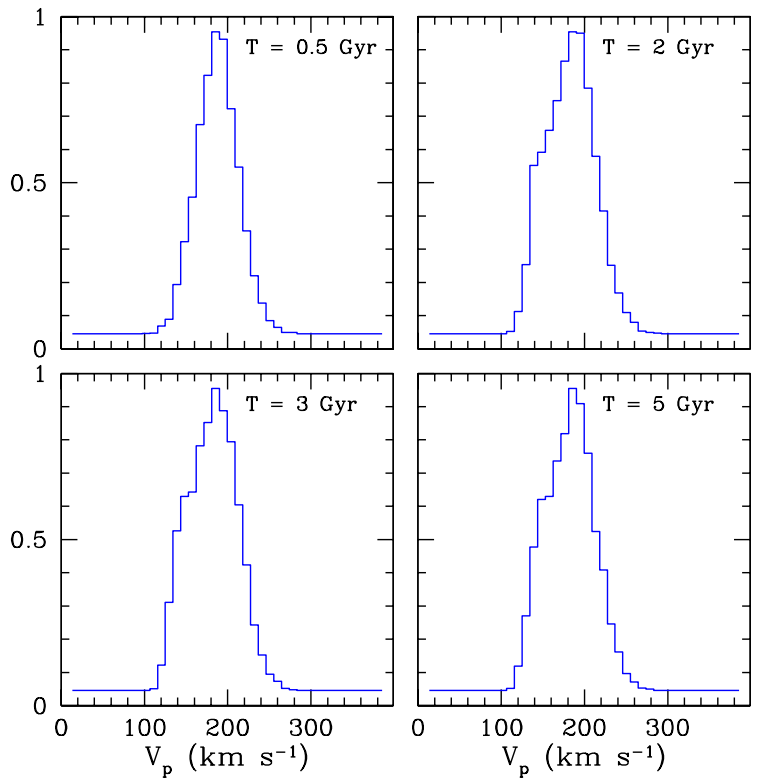

FIG. 3.- Probability distributions for the peculiar velocity of XTE J1118+ 480 at different disk crossings as obtained from the integration of 10000 orbits backward in time, where the current velocity components are drawn randomly from Gaussian distributions centered around the observed values. The distributions at different disk crossings do not differ significantly. This property allows the determination of an average mean peculiar velocity at the moment of the ejection from the Galactic disk which is independent of the exact time of the ejection.

grated the orbit of the system backward in time in the Galactic potential and derived the peculiar velocity of the binary center of mass at different disk crossings. As shown in $\S 3.1$, the average peculiar velocity of the binary computed over all the disk crossings between 0.5 and 5 Gyr ago is
$<V_{p}>=183 \pm 31 \mathrm{~km} \mathrm{~s}^{-1}$. We now explore the possibility that XTE J1118+480 acquired its high peculiar velocity as a result of a symmetric kick. The maximum symmetric kick compatible with the survival of the binary as a function of the orbital period after circularization is shown in Fig. 4. It is obtained when the mass ejected in the explosion is maximum, $\Delta M=M_{\mathrm{bh}}+m$. The three regions refer to values of the donor star mass $m$ of $1.2,1.5$ and $1.8 \mathrm{M}_{\odot}$, respectively, from bottom to top. For each value of $m$, the black hole mass vary in the range $6.0-8.0 \mathrm{M}_{\odot}$. The orbital period of the circularized binary is allowed to vary between a minimum value, corresponding to the case where the companion star fills its Roche Lobe and the system comes into contact after recircularization, and a maximum value set by the constraint that the donor star fills its Roche lobe within its lifetime because of magnetic braking (Kalogera 1999). The maximum kick velocity obtainable with a symmetric supernova explosion is then $V_{\text {sym,max }} \simeq 120 \mathrm{~km} \mathrm{~s}^{-1}$, not enough to impart a recoil to XTE J1118+480 as large as the observed peculiar velocity. In fact, the distribution of the peculiar velocity is such that there is a very low probability ( $0.9 \%)$ for $V_{p} \leq 120 \mathrm{~km} \mathrm{~s}^{-1}$ at a disk crossing (see Fig. 3). In particular, for the reference case $\left(m=1.5 \mathrm{M}_{\odot}, M_{\mathrm{bh}}=7 \mathrm{M}_{\odot}\right), V_{\mathrm{sym}, \max } \simeq 100 \mathrm{~km} \mathrm{~s}^{-1}$.

\subsection{Asymmetric kick}

The considerations of the $\S 3.2$ indicate that an asymmetric kick is likely to be needed in addition to a symmetric kick to produce the large space velocity of XTE J1118+480. A lower limit on the value of the asymmetric kick imparted to the system can be obtained if the direction of the orbital angular momentum vector of the system is known. Estimates of the inclination of the orbital plane of the binary relative to the line of sight are available from optical spectroscopy and photometry combined with theoretical models, giving $60^{\circ} \lesssim i \lesssim 80^{\circ}$ (Wagner et al. 2001, Frontera et al. 2001). We will consider an intermediate $i=70^{\circ}$ as a reference value. Unfortunately, the position angle $\Omega$ of the line of nodes with respect 


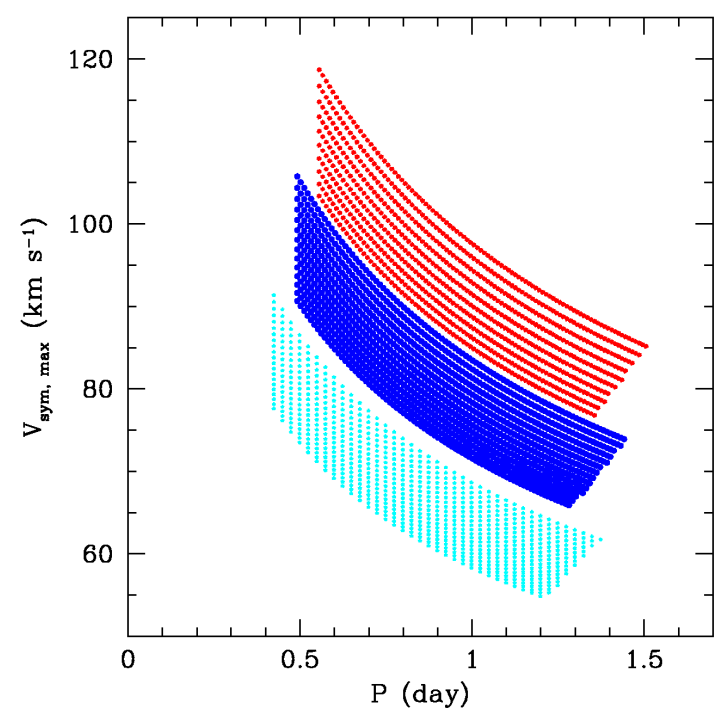

FIG. 4.- Maximum recoil velocity imparted to the binary center of mass in a symmetric supernova explosion as a function of the orbital period after the recircularization phase. The minimum value for the orbital period is set by the condition that the companion star fills its Roche lobe after recircularization while the maximum value is set by the constraint that the donor star fills its Roche lobe within its lifetime because of magnetic braking. The three regions refer to values of the donor star mass $m$ of $1.2,1.5$ and $1.8 \mathrm{M}_{\odot}$, respectively, from bottom to top. For each value of $m$, the black hole mass vary in the range $6.0-8.0 \mathrm{M}_{\odot}$.

to the celestial coordinates is not known and therefore the exact 3D-orientation of the orbital plane of the binary can not be reconstructed. However, the components of the angular momentum vector can be written as a function of $i$ and $\Omega$. If we randomly draw values for the undetermined angle from a uniform distribution in the range $[0-2 \pi)$, we can compute at each disk crossing - the component $V_{p, \perp}$ of the peculiar velocity of XTE J1118+480 which is perpendicular to its orbital plane. Since the symmetric kick lies in the orbital plane while the asymmetric kick can have any direction, the component $V_{p, \perp}$ provides a lower limit for the magnitude of the asymmetric kick, once corrected for the contribution of the random motion of the binary's progenitor (about $10 \mathrm{~km} \mathrm{~s}^{-1}$ for one component).

Fig. 5 reports the distribution for $V_{p, \perp}$ at disk crossing from the 10000 Monte Carlo realizations which are closer to some specified times in the past, namely $0.5 \mathrm{Gyr}$ (the likely lower limit for $\tau_{\mathrm{ex}}$ ) and 2, 3, 5 Gyr ago, corresponding to the upper limit of $\tau_{\mathrm{ex}}$ for a companion star with initial mass of 1.8, 1.5 and $1.2 \mathrm{M}_{\odot}$ respectively. These histograms have broad peaks covering a range in $V_{p, \perp}$ from 60 to $140 \mathrm{~km} \mathrm{~s}^{-1}$. They show that the hypothesis of a null intrinsic kick in the direction perpendicular to the orbital plane has a probability of at most $5 \%$. Such probability corresponds to all the Monte Carlo realizations of the motion of XTE J1118+480 in the Galaxy for which $V_{p, \perp}$ at disk crossing is less than the unidimensional dispersion velocity of the progenitor of the binary system.

The distributions of $V_{p, \perp}$ presented in Fig. 5 are wider and flatter than those of $V_{p}$ (Fig. 3) since they result from the overlap of the distributions obtained for any possible value of $\Omega$ (in the upper panel of Fig. 6 we display a set of these distributions, for the case $\tau_{\mathrm{ex}}=3 \mathrm{Gyr}$ ago). The dominant source of indetermination arises from the uncertainties on the current

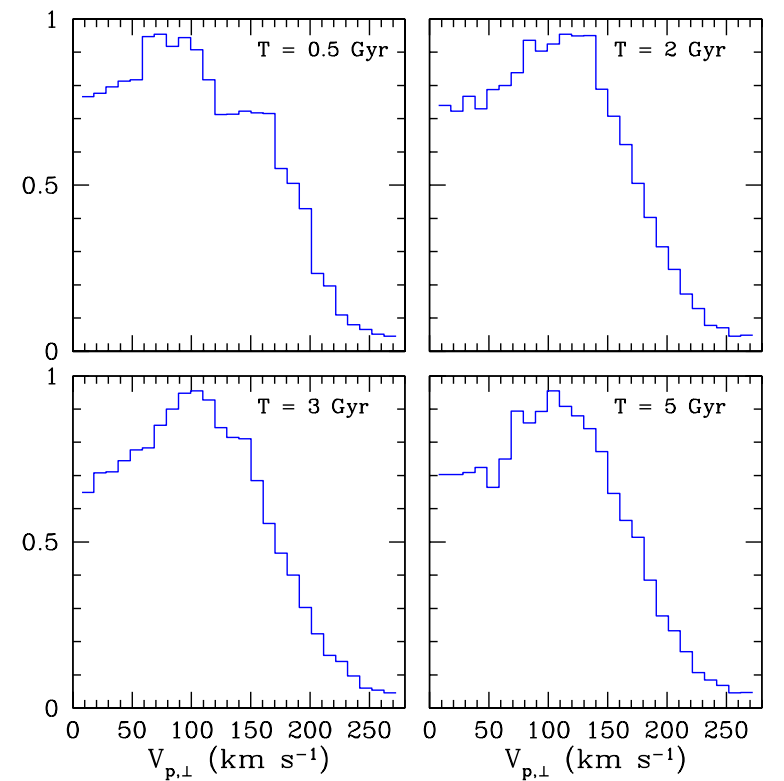

FIG. 5.- Probability distributions for the component of the peculiar velocity of XTE J1118+480 which is perpendicular to its orbital plane. The histograms are obtained with the orbit integrator described in $\S 3.1$ assuming an inclination angle of the orbital plane $i=70^{\circ}$. Distributions at different disk crossings, between 0.5 and 5 Gyr ago, are represented.

kinematic parameters of XTE J1118 +480 and on the time of occurrence of the supernova event $\tau_{e x}$. As an example, comparison of the upper and central panels of Fig. 6 shows that even the knowledge of $\Omega$ with a $10^{\circ}$ accuracy could not ensure a significant determination of $V_{p, \perp}$, unless the errors on the measured radial and transverse velocity of XTE J1118+480 were reduced down to about $1 \%$. In particular, the large uncertainties on the radial velocity $\left(V_{r}=26 \pm 17 \mathrm{~km} \mathrm{~s}^{-1}\right.$, McClintock et al. $2001 ; 15 \pm 10 \mathrm{~km} \mathrm{~s}^{-1}$, Wagner et al. 2001) sensibly affect the global error on the system's space velocity. The lower panel in Fig. 6 displays the effect of the indetermination on $\tau_{e x}$ : the distributions of $V_{p, \perp}$ summed over all the disk passages between 0.5 and $3 \mathrm{Gyr}$ ago have broader peaks than those corresponding to the single passage at about $3 \mathrm{Gyr}$ ago.

\section{CONCLUSIONS}

We have investigated the origin of the large peculiar velocity and high-latitude orbit of the soft X-ray transient XTE J1118+480. We constrain the origin of the binary system by studying the evolutionary state of the companion star and by calculating the orbital trajectory of the binary system in the potential of the Galaxy.

Based on the high CNO enrichment of the donor star, we argue that it was born as a $1.2-1.8 \mathrm{M}_{\odot}$ main-sequence star, with a most probable value of $1.5 \mathrm{M}_{\odot}$ (Haswell et al 2002). The turn-off age of such a star is between 1 and $4.5 \mathrm{Gyr}$. The subsequent evolution as a semi-detached binary lasts for about $0.8 \mathrm{Gyr}$, providing a tentative upper limit to the age of the binary system of about 2 to $5.5 \mathrm{Gyr}$, which is much smaller than the typical age of globular clusters. We therefore conclude that the binary cannot have formed in a globular cluster, but must have formed in the Galactic disk.

In that case, the binary must have been propelled in its current galactic high latitude orbit by the supernova explosion 

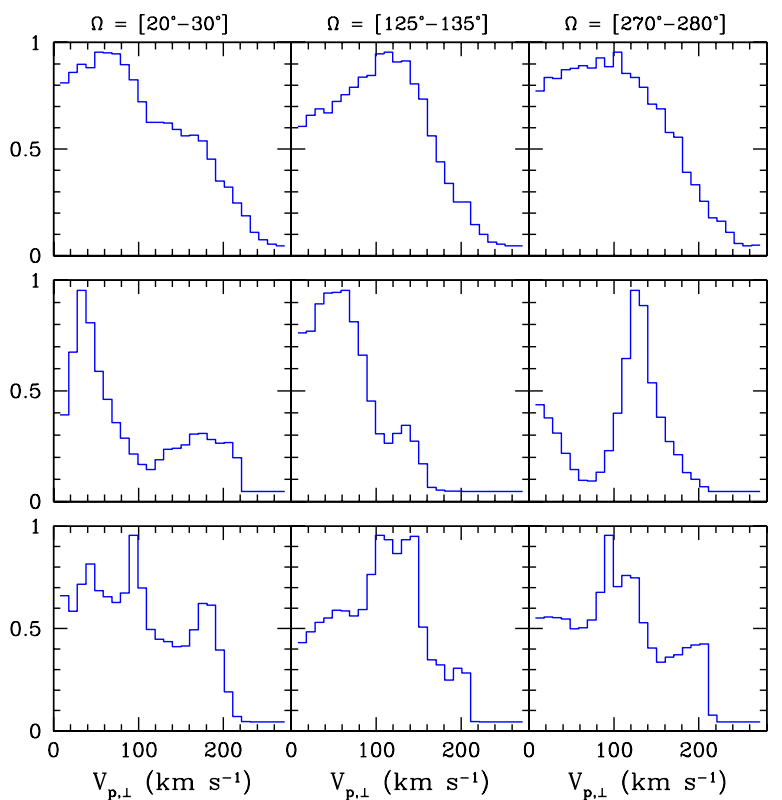

FIG. 6.- Probability distributions for the component of the peculiar velocity of the binary perpendicular to the orbital plane. The histograms are obtained with the orbit integrator described in $\$ 3.1$ assuming an inclination angle of the orbital plane $i=70^{\circ}$. The distributions in the upper panel are for a supernova event occurred $\tau_{\mathrm{ex}}=3 \mathrm{Gyr}$ ago in the Galactic disk and for different possible ranges of values of the position angle of the line of the node $\Omega$. The distributions in the central panel are for the same parameters but reducing of a factor 10 the error bars on the available determination of the current 3D-velocity of the system. The distributions in the lower panel are as in the central panel but summed over all the disk crossings between 0.5 and $3 \mathrm{Gyr}$ ago.

in which the black hole formed. This must have happened shortly after the formation of the binary as the progenitor of the black hole lives very short compared to the companion star.

We calculate the orbital trajectory of the binary backward in time through the potential of the Galaxy for a time comparable to the age of the system to find the average peculiar velocity of the system at the location where it was ejected about $0.5-$ $5 \mathrm{Gyr}$ ago. From these calculations we conclude that, upon birth, the binary system must have received an average kick of $183 \pm 31 \mathrm{~km} \mathrm{~s}^{-1}$.

The maximum systemic velocity which can be acquired by the binary upon symmetric mass loss is about $100-$ $120 \mathrm{~km} \mathrm{~s}^{-1}$. Therefore, a symmetric supernova is not likely (on a $2.7 \sigma$ level) to have propelled the binary in its current orbit but an additional asymmetric kick is required. Using the available information on the $3 \mathrm{D}$ orientation of the binary, we derive a probability of $95 \%$ for the component of the kick perpendicular to the orbital plane to exceed the random vertical motion in the Galactic disk. The average of this distribution is $93_{-60}^{+55} \mathrm{~km} \mathrm{~s}^{-1}$. These results are rather insensitive to the exact age of the system but depends heavily on the kinematic parameters of the system. The measurements of the spatial velocity of the binary should be ten times more accurate than currently available in order to draw tight values on the asymmetric kick.

\section{ACKNOWLEDGMENTS}

We would like to thank Jasinta Dewi for performing binary evolutionary calculations relevant for this work, Franca D'Antona for useful discussions and Gijs Nelemans and Tom Maccarone for useful comments on the manuscript. This work was supported by the Netherlands Organization for Scientific Research (NWO), the Royal Netherlands Academy of Arts and Sciences (KNAW), the Netherlands Research School for Astronomy (NOVA) and the Italian Ministry of University and Research (MIUR) under the national program Cofin 2003".

\section{REFERENCES}

Bhattacharya, D. \& van den Heuvel, E.P.J., 1991, Physics Rep. 203, 1

Blaauw, A., 1961, BAN 15, 165

Burrows, A. \& Hayes, J. 1996, Phys. Rev. Lett., 76, 352

Campana, S., Colpi, M., Mereghetti, S., Stella, L., \& Tavani, M., 1998, A\&A. Rv., 8, 279

Colpi, M. \& Wasserman, I. 2002, ApJ, 581, 1271

Cook, L., Patterson, J., Buczynski, D., \& Fried, R., 2000, IAU Circ, 7397

Cordes, J.M. , Romani, R.W., \& Lundgren, S.C., 1993, Nature, 362, 133

Frontera, F. et al., 2001, ApJ, 561, 1015

Haswell, C.A., Hynes, R.I., King, A.R., \& Schenker, K., 2002, MNRAS, 332, 928

Heger, A., Langer, N., \& Woosley, S.E. 2000, ApJ, 528, 368

Israelian, G., Rebolo, R., Basri, G., Casares, J., \& Martín, E.L., 1999, Nature, 401,142

Kalogera, V., 1999, ApJ, 521, 723

Kulkarni, S.R., Hut, P, \& McMillan, S., 1993, Nature, 364, 421

Lai, D., Chernoff, D.F., \& Cordes, J.M. 2001, ApJ, 549, 1111

Lee, C.H., Brown, G.E., \& Wijers, R.A.M.J., 2002, ApJ, 575, 996

Liu, Q.Z., van Paradijs, J.,\& van den Heuvel, E.P.J., 2001, A\&A, 368, 1021

McClintock, J., Garcia, M., Caldwell, N., Falco, E.E., Garnavich, P.M., \& Zhao, P., 2001, ApJ, 551, L147
Mihalas, D. \& Binney, J., 1981, Galactic Astronomy, Structure and Kinematics (New York: Freeman)

Mirabel, I.F., Dhawan, V., Mignani, R.P., Rodrigues, I., \& Guglielmetti, F., 2001, Nature, 413, 139

Mirabel, I.F., Mignani, R.P., Rodrigues,I., Combi, J.A., Rodriguez, L.F., \& Guglielmetti, F., 2002, A\&A, 395, 595

Nelemans, G., Tauris, T.M., \& van den Heuvel, E.P.J., 1999, A\&A,352, L87

Orosz, J.A., Groot, P.J., van der Klis, M., McClintock, J.E., Garcia, M.R.,

Zhao, P., Jain, R.K., Bailyn, C.D., \& Remillard, R.A., 2002, ApJ, 568, 845 Paczynski, B., 1990, ApJ, 348, 485

Pols, O.R., Tout, C.A., Eggleton, P.P., \& Zhanwen, H., 1995, MNRAS, 274, 964

Portegies Zwart, S.F., Verbunt, F., \& Ergma, E., 1997, A\&A, 321, 207

Portegies Zwart, S.F. \& McMillan, S.L.W., 2000, ApJ, 528, L17

Sigurdsson, S. \& Hernquist, L. 1993, Nature, 364, 423

Tanaka, Y. \& Shibazaki, N., 1996, ARA\&A, 34, 607

Wagner, R.M., Foltz, C.B., Shahbaz, T., Casares, J., Charles, P.A., Starrfield, S.G., \& Hewett, P., 2001, ApJ, 556, 42

White, N.E. \& van Paradijs, J., 1996, ApJ, 473, L25 